\title{
NUMERICAL SIMULATION OF THE EFFECT OF DAMAGED INDUCED SOFTENING ON THE INFLATION OF A CIRCULAR RUBBER MEMBRANE
}

\author{
ALAN S. WINEMAN \\ Department of Mechanical Engineering and Applied Mechanics, The University of Michigan, \\ Ann Arbor, Michigan 48109, U.S.A. \\ and \\ HUGH E. HUNTLEY \\ Department of Mechanical Engineering, University of Michigan-Dearborn, Dearborn, \\ Michigan 48128, U.S.A.
}

(Received 24 September 1993 ; in revised form 10 February 1994)

\begin{abstract}
Rubbery materials can undergo deformation induced microstructural changes leading to softening of response and permanent set. In this work, these materials are modeled by a constitutive equation that incorporates the following micromechanism: when the deformation becomes large enough, network junctions break during further increments of deformation and then heal. New networks are formed by this conversion process, each unstressed in the local configuration at which it is formed. The constitutive equation is used to describe the response of the particles of an initially flat circular membrane which is inflated by lateral pressure. The original material and each newly formed network are assumed to respond as neo-Hookean elastic materials. Results of a numerical simulation show the influence of this conversion process on the distribution of stretch ratios, inflated shapes and the relation between the pressure and the crown stretch ratio.
\end{abstract}

\section{INTRODUCTION}

The general form of the constitutive equation for non-linear elastic solids is based on assumptions which imply that stress arises from a single unchanging material micromechanism at all stages of deformation. However, rubber-like materials exhibit changes in micromechanism evidenced by the occurrence of permanent set under large deformations (Hart-Smith, 1966). Rajagopal and Wineman (1992) have presented a constitutive theory which can be used to model such mechanical response. In their model, the stress is determined by one micromechanism within some regime of deformation; as deformation increases, a new micromechanism arises which affects the mechanical response. They considered the particular example in which material acts as a rubbery solid if the deformations are not too large. When deformations become sufficiently large, network junctions in the original material break and then reform to produce a new network with a new unstressed local configuration. Their work allowed for continuous conversion of the original material to new networks as deformation proceeds. It was shown that the material can undergo substantial softening, and that there is permanent set when the stress is removed.

There have been several applications of this constitutive theory to problems involving non-homogeneous deformations. The examples all assume that the material is incompressible and that new networks are generated at sufficiently large deformations. Wineman and Rajagopal (1990) studied the finite extension and torsion of a circular cylinder. They showed that as the angle of twist increases, there evolves an inner core of material which consists of the original network and an outer shell of the modified material. These regions are separated by an interface whose radius is determined by the angle of twist. They also studied the influence of the material response on the torque-twist relation.

Huntley (1992) considered the problem in which a hollow concentric cylinder is fixed at its inner surface and its outer surface is rotated about the centerline. Each particle of the cylinder is subjected to a simple shear deformation, the magnitude of which decreases with 
increasing radius. As the rotation increases, the cylinder develops an inner core of multinetwork material and an outer shell of original material. They are separated by an interface whose radius can be determined from the shear response of the original material and the applied moment.

Huntley (1992) also analysed the radial expansion of thick-walled hollow spheres under either internal pressure or external radial tension. Each particle is subjected to equal biaxial extension, with the stretch ratio decreasing with the radius. As in the other examples, there are two regions of material response. There is an inner spherical shell of multi-network material and an outer shell of original material. The radius of the interface is determined by the radius of the inner or outer surface. Huntley determined the influence of the material response on the pressure-radius relation of the hollow sphere.

The present work is concerned with a thin uniform circular sheet of material which is fixed at its boundary, and which is subjected to a uniform pressure over one of its surfaces. The sheet inflates into an axially symmetric bubble in which each particle can be regarded as being in a state of unequal biaxial extension. It is expected that the crown region consists of multi-network material, while the support region consists of the original material. There are several reasons for studying this membrane inflation problem. First, it simulates a possible experiment. Indeed, the inflation of a circular membrane has been used in nonlinear elasticity in conjunction with the determination of material properties. Treloar (1944) measured profiles of the inflated sheet. Adkins and Rivlin (1952) calculated the profiles using a measured strain energy density function in order to compare their results with the data of Treloar. Hart-Smith and Crisp (1967) and Wineman et al. (1979) each proposed methods for using the measured profile to determine the strain energy function in nonlinear elasticity. It may be possible to use this experiment as a means of measuring properties in the constitutive theory considered here. Second, in the examples mentioned above, the interface lies in the interior of a cylinder or a sphere. The membrane configuration provides a means of directly observing the location of the interface. Third, the problem requires a more sophisticated method of solution for the deformation than in the previous examples. Finally, when the material response is non-linear elastic, the pressure-membrane height relation usually has a local maximum. It is interesting to determine the effect on this maximum for the material response considered here. In this work then, consideration is given to the development and application of a new constitutive equation, issues that arise in carrying out a numerical solution, and implications of this material model for the response of the structure.

The constitutive equation is presented in Section 2 and the equations governing the response of the inflated membrane are presented in Section 3. Section 4 contains the formulation of the boundary value problem for the inflated shape of the membrane. This is solved by the numerical method outlined in Section 5. Results for a numerical example are discussed in Section 6.

\section{CONSTITUTIVE EQUATION}

Consider a body which is homogeneous, initially stress free and which undergoes homogeneous deformations. Let $\kappa(0)$ denote the configuration of a body in its initial undeformed stress free state, which is taken as a reference configuration, and let $\kappa(t)$ denote its configuration at a later time $t$. The deformation gradient of configuration $\kappa(t)$ with respect to configuration $\kappa(\tau)$ is denoted as $\mathbf{F}_{\tau}^{t}$.

It is assumed that there is a regime of deformations from the reference configuration $\kappa(0)$ in which the mechanical response is that of an incompressible, isotropic, non-linear elastic solid. Let $\mathbf{F}_{0}=\mathbf{F}_{0}^{t}$, a deformation gradient within this regime. The constitutive equation has the form

$$
\sigma=-p \mathbf{I}+\phi_{1} \mathbf{B}_{0}+\phi_{-1} \mathbf{B}_{0}^{-1}
$$

where $\mathbf{B}_{0}=\mathbf{F}_{0} \mathbf{F}_{0}^{\mathrm{T}}, I_{1}$ and $I_{2}$ are the principal invariants of $\mathbf{B}_{0}, W\left(I_{1}, I_{2}\right)$ is the Helmholtz 
free energy density function, $\phi_{1}=2 \partial W / \partial I_{1}, \phi_{-1}=-2 \partial W / \partial I_{2}$ and $p$ is the arbitrary scalar arising from the incompressibility constraint.

This response can be regarded as governed by a single material micromechanism, the reconfiguration of macromolecules. It is assumed that there is a set of deformations at which a new micromechanism is activated. Further deformation then leads to changes in the microstructure of the material. For convenience in presentation, attention is focused on the specific micromechanism of scission of network junctions, i.e. cross-links or entanglements, and their subsequent reforming into new networks with new unstressed local configurations. [For further discussion, see Rajagopal and Wineman (1992).]

In order to describe this process, let a scalar parameter $s$ be defined which is associated with configuration $\kappa(t)$ by the relation

$$
s=\Sigma\left(\mathbf{F}_{0}\right)
$$

Parameter $s$ is called the deformation state parameter. At the initial configuration, $t=0$, $\mathbf{F}_{0}=I$ and $s=0$. The value of $s$ increases as the deformation becomes larger, and decreases as the deformation is reduced. For deformation histories which are described by a single parameter, such as uniaxial extension, equal biaxial deformation and simple shear, it is clear what is meant by an increasing or decreasing deformation. For more general deformation histories, there is no unique definition of what is meant by the deformation becoming "larger" or "smaller". This depends on the particular deformation process under consideration. Relation (2) establishes a correspondence between time $t$ and the deformation state parameter $s$.

It is assumed that there is a set of deformation gradients $\mathbf{F}_{0}$ corresponding to configurations at which the new micromechanism is activated. This event is characterized by introducing a scalar-valued activation function $A\left(\mathbf{F}_{0}\right)$. The new micromechanism is said to be activated when a deformation gradient of the set satisfies the activation criterion,

$$
A\left(\mathbf{F}_{0}\right)=0 .
$$

Let each deformation gradient of this set have the same value of the deformation state parameter $s$, denoted by $s_{\mathrm{a}}$. Then the activation function is defined by

$$
A\left(\mathbf{F}_{0}\right)=\Sigma\left(\mathbf{F}_{0}\right)-s_{\mathrm{a}}
$$

Material frame indifference, isotropy and incompressibility imply that functions $A$ and $\Sigma$ depend on $\mathrm{F}_{0}$ through its invariants $I_{1}$ and $I_{2}$. These functions are now denoted by $A\left(I_{1}, I_{2}\right)$ and $\Sigma\left(I_{1}, I_{2}\right)$, where the same symbols have been retained for notational simplicity.

Consider a sequence of deformations which increase from the initial state, while the corresponding values of the parameter $s$ increase monotonically from the value $s=0$. By (2), there is a one-to-one correspondence between the times $t$ and the values of $s$ during this sequence. It is convenient to utilize this correspondence and introduce a change from time parameter $t$ to deformation state parameter $s$. Thus, retaining the same notation, $\kappa(s)$ now denotes the configuration corresponding to deformation state parameter $s . \mathbf{F}_{0}^{s}$ denotes the deformation gradient of the configuration at the current state $s$ with respect to the configuration at the initial state $s=0$ and is denoted by $\mathbf{F}_{0}=\mathbf{F}_{0}^{s}$. Also, introduce the deformation gradient $\mathbf{F}_{s}=\mathbf{F}_{s}^{s}$, which represents the deformation gradient of the configuration at the current state $s$ with respect to the configuration at state $\hat{s}$.

During each increment in the sequence of increasing deformation from activation, a certain volume fraction of network junctions of the original material is broken. This fraction depends on the extent of deformation of the original material. The newly broken network junctions then immediately reform to produce a new undistorted network. During further deformation, this newly formed network deforms and contributes to the total stress. It is assumed, for the sake of simplicity, that there is no scission of newly formed networks. The material, as it undergoes this process of microstructural transformation, is said to be 
converting. In the remainder of this article, the terminology "network" is generalized to refer to either the original material or any newly transformed material.

The total stress at each stage of deformation is defined to be the superposition of contributions from the remaining portion of the original material and from each network formed during the deformation process. Thus, if $\sigma$ is the Cauchy stress at the deformation state corresponding to state parameter $s$,

$$
\sigma=-p \mathrm{I}+\sigma^{\mathrm{R}}+\int_{s_{\mathrm{a}}}^{s} a(\hat{s}) \sigma^{\mathrm{N}} \mathrm{d} \hat{s}
$$

where: (1) $p$ is an arbitrary scalar arising from the constraint of incompressibility; (2) $\sigma^{\mathrm{R}}$ is the stress in the remaining original material; (3) $a(\hat{s}) \mathrm{d} \hat{s}$ is the volume fraction of converted material formed during the interval of deformation as the state parameter increases from $\hat{s}$ to $\hat{s}+\mathrm{d} \hat{s} ;(4) \sigma^{\mathrm{N}}$ is the stress per unit volume in the network formed at $\hat{s}$. The latter depends on the deformation gradient $F_{s}$, which is the gradient of the current configuration with respect to the configuration of the material that formed at $\hat{s}$.

Let $b(s)$ denote the volume fraction of the remaining portion of the original material at state $s$. Then $\sigma^{\mathrm{R}}$ has the form

$$
\sigma^{\mathrm{R}}=b(s)\left[\phi_{1} \mathbf{B}_{0}+\phi_{-1} \mathbf{B}_{0}^{-1}\right]
$$

For simplicity, the rate of decrease of volume fraction of original material is assumed to equal the rate of increase of volume fraction of material with new microstructure. This implies that

$$
b(s)=1-\int_{s_{\mathrm{a}}}^{s} a(\hat{s}) \mathrm{d} \hat{s}
$$

The new network formed at $\hat{s}$ responds as an incompressible non-linear isotropic elastic material. Its configuration at $\hat{s}$ is taken as a stress free configuration. For simplicity of modeling, the mechanical response is assumed to be the same for each new network. Thus

$$
\sigma^{\mathrm{N}}=\bar{\phi}_{1} \mathbf{B}_{s}+\bar{\phi}_{-1} \mathbf{B}_{s}^{-1}
$$

where $\mathbf{B}_{s}=\mathbf{F}_{s} \mathbf{F}_{s}^{\mathrm{T}}$, and $\bar{\phi}_{1}, \bar{\phi}_{-1}$ are material property functions which depend on the invariants of $\mathbf{B}_{\hat{s}}$. On combining (5), (6) and (8), the constitutive equation during the process of conversion as deformation increases beyond activation takes the representation

$$
\sigma=-p \mathbf{I}+b(s)\left[\phi_{1} \mathbf{B}_{0}+\phi_{-1} \mathbf{B}_{0}^{-1}\right]+\int_{s_{\mathbf{a}}}^{s} a(\hat{s})\left[\bar{\phi}_{1} \mathbf{B}_{s}+\bar{\phi}_{-1} \mathbf{B}_{s}^{-1}\right] \mathrm{d} \hat{s} .
$$

It is further assumed that at a typical state of deformation corresponding to $s^{*}>s_{\mathrm{a}}$, there exists a sequence of deformations for which the state parameter decreases and no additional microstructural transformation occurs. Then, $a(s)=0$, and the upper limit of the integral in (9) is fixed at $s^{*}$. Moreover, (7) shows that $b(s)$ is fixed at the value $b\left(s^{*}\right)$. Equation (9) becomes

$$
\sigma=-p \mathbf{I}+b\left(s^{*}\right)\left[\phi_{1} \mathbf{B}_{0}+\phi_{-1} \mathbf{B}_{0}^{-1}\right]+\int_{s_{\mathrm{a}}}^{s^{*}} a(\hat{s})\left[\bar{\phi}_{1} \mathbf{B}_{s}+\bar{\phi}_{1} \mathbf{B}_{s}^{-1}\right] \mathrm{d} \hat{s}
$$


The body under consideration is initially a thin disk of uniform thickness $2 h_{0}$ and radius $R_{0}$, with $h_{0} / R_{0} \ll 1$. If $(R, \Theta, Z)$ denote the coordinates of a cylindrical coordinate system, the body initially occupies the region defined by

$$
0 \leqslant R \leqslant R_{0}, \quad 0 \leqslant \Theta<2 \pi, \quad-h_{0} \leqslant Z \leqslant h_{0}
$$

It is assumed that each particle of the disk is in an initial undeformed stress free configuration, and that the particles are identical. The mechanical response of each particle, as it undergoes a local homogeneous deformation, is described by the constitutive model in Section 2.

The particles on the surface $R=R_{0}$ are fixed to a rigid boundary. The sheet is assumed to undergo axisymmetric inflation caused by uniform pressure $q_{0}$ applied to the surface $Z=-h_{0}$. The surface $Z=h_{0}$ remains free of traction. As the sheet inflates, each particle undergoes a different local homogeneous deformation history. During this process, some particles may undergo conversion. In order to calculate the stresses, it is necessary to track the local configuration history of each particle. The method for accomplishing this is described in Section 4.

When the pressure $q_{0}$ is sufficiently small, each particle responds in its non-linear elastic regime. Since $h_{0} / R_{0} \ll 1$, the deformed state can be approximated using the theory of nonlinear elastic membranes [see Green and Adkins (1960)]. The midsurface $Z=0$ in the reference state deforms into a surface of revolution. According to the membrane theory, line segments which are perpendicular to the midsurface in the reference state can be regarded as remaining straight and perpendicular to the surface in its deformed state. The variation of kinematic quantities through the thickness of the deformed membrane can be neglected. That is, the deformed shape of a surface $Z=$ constant is approximately the same as the deformed shape of the surface $Z=0$. Consider the stress components acting on surfaces perpendicular to the midsurface. The variation of these stresses through the thickness can also be neglected. Consider a surface $Z=$ constant. The stress components acting on the deformed state of such a surface can be neglected compared to the other stress components. In effect, it is sufficient to consider quantities associated with the deformed midsurface. Only their variation over the midsurface need be determined.

Examples involving neo-Hookean and Mooney-Rivlin materials [e.g. Green and Adkins (1960)], show that the stretch is greatest at the axis of symmetry of the inflated sheet, i.e. its "crown", and decreases monotonically toward the outer support. Thus, the deformation state parameter $s$, which increases with the deformation, can be expected to be greatest at the axis of symmetry and decrease toward the support. The particle at the crown is the first to undergo activation and conversion. During inflation, the sheet then consists of a central region of material undergoing conversion and an outer region of elastic material.

It is assumed that the membrane approximation remains valid during the conversion process. Consider the particles on a cylindrical surface of radius $R$ in the reference configuration. All such particles have approximately the same deformed state, and hence approximately the same value of the deformation state parameter. Thus conversion is assumed to occur simultaneously at all particles on that surface. During the conversion process, these particles are at the same state and hence have the same value of the deformation state parameter. As in the case of an elastic membrane, kinematic quantities vary only over the midsurface.

\section{Kinematics of deformation}

Equations for the kinematics of deformation and for equilibrium, incorporating the membrane approximation, are now presented. They are analogous to those for a non-linear viscoelastic membrane [see Wineman (1976), where additional details can be found]. 
The coordinates of a particle $P$ on the midsurface of the reference configuration are $(R, \Theta, 0)$. The radius $R$ of a particle acts as its label. If $(r, \theta, z)$ are the coordinates of particle $P$ in an inflated state, then

$$
\begin{aligned}
& r=r(R), \\
& \theta=\Theta, \\
& z=z(R) .
\end{aligned}
$$

$r(R)$ and $z(R), R \in\left[0, R_{0}\right]$, describe the inflated shape of the midsurface. They also depend on a parameter, each value of which is associated with a fixed state of inflation. This inflation parameter is introduced below. The quantities and equations presented in the remainder of this section apply over the midsurface for each fixed state of inflation, that is for each fixed value of the inflation parameter. For the purpose of notational brevity, this parameter is suppressed until it is needed.

It is a consequence of the axisymmetry of the deformation that the circumferential, meridional and normal directions, relative to the deformed state of the midsurface, are principal directions of stretch. Using the notation introduced in Section $2, \lambda_{0}$ denotes the ratio of the current length of a line element to its length in the initial configuration. $\left(\lambda_{0}\right)_{i}$, $i=1,2,3$, denotes a principal stretch ratio. For notation convenience, we now let $\lambda_{i}=\left(\lambda_{0}\right)_{i}$. The stretch ratios are:

in the meridional direction,

$$
\lambda_{1}=\left[\left(\frac{\partial r}{\partial R}\right)^{2}+\left(\frac{\partial z}{\partial R}\right)^{2}\right]^{1 / 2}
$$

in the circumferential direction,

$$
\lambda_{2}=\frac{r}{R}
$$

in the normal direction, by incompressibility,

$$
\lambda_{3}=\frac{1}{\lambda_{1} \lambda_{2}}
$$

\section{Equilibrium equations}

The principal directions of stretch are also principal directions of stress, due to material isotropy. Consider a material element bounded by surfaces whose normal vectors are in the meridional and circumferential directions in the deformed midsurface, and in the direction normal to the midsurface. Let the normal stresses on these surfaces be denoted by $\sigma_{1}, \sigma_{2}, \sigma_{3}$, respectively. According to the assumptions of membrane theory, $\left|\sigma_{3} / \sigma_{1}\right| \ll 1$, $\left|\sigma_{3} / \sigma_{2}\right| \ll 1$. Hence, each particle is in a state of plane stress.

The differential equation of equilibrium in the meridional direction is

$$
\frac{\partial\left(\lambda_{3} \sigma_{1}\right)}{\partial R}+\frac{\eta \lambda_{3}\left(\sigma_{1}-\sigma_{2}\right)}{\lambda_{2} R}=0
$$

where

$$
\eta=\frac{\partial r}{\partial R}
$$

and the equation has been transformed to apply in the reference configuration. The equation of equilibrium in the normal direction at each point on the midsurface is 


$$
\kappa_{1} \lambda_{3} \sigma_{1}+\kappa_{2} \lambda_{3} \sigma_{2}=\frac{q_{0}}{h_{0}}
$$

where $\kappa_{1}$ and $\kappa_{2}$ are principal curvatures defined by

$$
\begin{gathered}
\kappa_{1}=\frac{\eta \frac{\partial \lambda_{1}}{\partial R}-\lambda_{1} \frac{\partial \eta}{\partial R}}{\lambda_{1}^{2}\left[\lambda_{1}^{2}-\eta^{2}\right]^{1 / 2}}, \\
\kappa_{2}=\frac{\left(\lambda_{1}^{2}-\eta^{2}\right)^{1 / 2}}{\lambda_{1} \lambda_{2} R},
\end{gathered}
$$

and $q_{0}$ is the pressure.

As shown by Green and Adkins (1960), an alternative to eqns (18)-(20) is given by

$$
2 h_{0} \lambda_{3} \sigma_{1} \kappa_{2}=q_{0}
$$

Using (20), this leads to the equation

$$
\eta= \pm\left[\lambda_{1}^{2}-\left(\frac{q_{0} \lambda_{1}^{2} \lambda_{2}^{2} R}{2 h_{0} \sigma_{1}}\right)^{2}\right]^{1 / 2}
$$

Because the membrane is being inflated, it is reasonable to assume that near $R=0$, $\eta=\partial r / \partial R>0$ and the positive sign applies in (22). In the examples presented by Green and Adkins, the inflated shape of the membrane can expand beyond the support so that for some particles it is possible that $r(R) \geqslant R_{0}$. In this case, $\eta=\partial r / \partial R<0$.

\section{Stress-stretch relations}

Consider an orthonormal coordinate system whose base vectors, at each point of the deformed membrane, are in the meridional, circumferential and normal directions. With respect to this system,

$$
\mathbf{B}_{s}=\operatorname{diag}\left(\frac{\lambda_{1}^{2}}{\hat{\lambda}_{1}^{2}}, \frac{\lambda_{2}^{2}}{\hat{\lambda}_{2}^{2}}, \frac{\lambda_{3}^{2}}{\hat{\lambda}_{3}^{2}}\right)
$$

where $\hat{\lambda}_{i}$ is a principal stretch ratio of a line element in the configuration at $\hat{s}$ with respect to its length in the initial configuration. Similarly,

$$
\mathbf{B}_{0}=\operatorname{diag}\left(\lambda_{1}^{2}, \lambda_{2}^{2}, \lambda_{3}^{2}\right)
$$

The invariants of $\mathbf{B}_{0}$ are

$$
\begin{aligned}
& I_{1}=\lambda_{1}^{2}+\lambda_{2}^{2}+\lambda_{3}^{2}, \\
& I_{2}=\lambda_{1}^{-2}+\lambda_{2}^{-2}+\lambda_{3}^{-2} .
\end{aligned}
$$

The deformation state parameter is taken to be a function of the radius in $I_{1}-I_{2}$ space, that is, eqn (2) has the form

$$
s=s\left(\left(I_{1}-3\right)^{2}+\left(I_{2}-3\right)^{2}\right)
$$

where the specific form will be given in Section 5. 
Consider the response when $s<s_{\mathrm{a}}$, i.e. when activation has not yet occurred. The original network at a particle is assumed to respond as a neo-Hookean material, so that $\phi_{1}=\mu^{0}$, a constant, and $\phi_{-1}=0$ in eqn (1). It follows from eqns (1), (24) and the membrane approximation $\sigma_{3}=0$, that

$$
\begin{aligned}
& \sigma_{1}=\mu^{0}\left(\lambda_{1}^{2}-\lambda_{3}^{2}\right) \\
& \sigma_{2}=\mu^{0}\left(\lambda_{2}^{2}-\lambda_{3}^{2}\right) .
\end{aligned}
$$

Next, consider the response when $s>s_{\mathrm{a}}$. As a particle undergoes conversion, each newly formed network is assumed to respond as a neo-Hookean material, so that in (9), $\bar{\phi}_{1}=\mu^{\mathrm{N}}$ a constant, and $\bar{\phi}_{-1}=0$. If the modulus of the remaining original material is denoted as $\mu^{0}=\mu^{\mathrm{R}}$ then, in general, $\mu^{\mathrm{R}} \neq \mu^{\mathrm{N}}$. It follows from (9), (23) and the membrane approximation $\sigma_{3}=0$ that

$$
\sigma_{\alpha}=h(s) \mu^{\mathrm{R}}\left(\lambda_{\alpha}^{2}-\lambda_{3}^{2}\right)+\int_{s_{\mathrm{a}}}^{s} a(\hat{s}) \mu^{\mathrm{N}}\left(\frac{\lambda_{\alpha}^{2}}{\hat{\lambda}_{\alpha}^{2}}-\frac{\lambda_{3}^{2}}{\hat{\lambda}_{3}^{2}}\right) \mathrm{d} \hat{s}, \quad \alpha=1,2
$$

\section{BOUNDARY VALUE PROBLEM}

The same approach is used here as was used by Wineman (1976) in the case of a viscoelastic membrane. A boundary value problem is established for the stretch ratios $\lambda_{1}$ and $\lambda_{2}$ and the associated kinematic quantity $\eta$, defined in eqn (17), at each inflated state. Once these have been found for an inflated state, the deformation (12) can be calculated from eqns (13) and (14). The stresses can be evaluated using eqns (27) and (28).

Smoothness assumptions on the deformation $r(R), z(R)$ imply that $\lambda_{1}=\lambda_{2}$ at $R=0$, at each inflated shape of the membrane. Let this common value be denoted by $t$, which is referred to as the crown stretch ratio. According to the example for neo-Hookean membranes in Green and Adkins (1960), the crown stretch ratio increases monotonically as the membrane becomes more highly inflated. On the other hand, the pressure $q_{0}$ initially increases, has a local maximum, and then decreases as the membrane inflation increases. The crown stretch ratio $t$ is therefore used to parametrize the inflated states. Thus,

$$
\begin{aligned}
\lambda_{1} & =\lambda_{1}(R, t), \\
\lambda_{2} & =\lambda_{2}(R, t), \\
\eta & =\eta(R, t) .
\end{aligned}
$$

According to eqns (25), (26) and (29), $s=s(R, t)$. For each value of $t, \lambda_{1}(R, t)$ and $\lambda_{2}(R, t)$ give the stretch ratio distribution and $s(R, t)$ gives the deformation state parameter distribution over the particles of the membrane. A given value of the deformation state parameter occurs at different particles $R$ at different inflation levels $t$. For a fixed particle $R$, these functions give the stretch ratio and deformation state parameter histories as $t$ increases.

It is assumed that for a fixed particle label $R$, there is an interval of values of $t$ for which $s(R, t)$ has an inverse denoted by $t=\tau(R, s)$. When this is used in (29), the stretch ratio is related to the deformation state parameters $s$, i.e. $\lambda_{\alpha}=\lambda_{\alpha}(R, \tau(R, s))$. Let $\hat{t}$ denote the value of the crown stretch ratio at a previous level of inflation, and let $\hat{s}$ denote the corresponding value of the deformation state parameter at particle $R$. Then, $\hat{\lambda}_{\alpha}=\lambda_{\alpha}(R, \tau(R, S))$. The stresses at a particle $R$ undergoing conversion are then calculated when this is used in eqn (28).

Let the following non-dimensional variables be introduced into eqns (16)-(22), (27) and (28): 


$$
\tilde{R}=\frac{R}{R_{0}}, \quad \tilde{r}=\frac{r}{R_{0}}, \quad \tilde{z}=\frac{z}{R_{0}}, \quad \tilde{\sigma}_{\alpha}=\frac{\sigma_{\alpha}}{\mu^{\mathrm{R}}}, \quad Q=\frac{q_{0} R_{0}}{\mu^{\mathrm{R}} h_{0}} .
$$

The " " notation is dropped for notational convenience. The form of eqns (16), (17), (19) and (20) are the same as before. $Q$ replaces $q_{0} / h_{0}$ in eqns (18) and (22). $\mu^{\mathrm{R}}$ no longer appears in (27) and (28) becomes

$$
\sigma_{\alpha}=b(s)\left(\lambda_{\alpha}^{2}-\lambda_{3}^{2}\right)+\int_{s_{\mathrm{a}}}^{s} a(\hat{s}) \mu\left(\frac{\lambda_{\alpha}^{2}}{\hat{\lambda}_{\alpha}^{2}}-\frac{\lambda_{3}^{2}}{\hat{\lambda}_{3}^{2}}\right) \mathrm{d} \hat{s}, \quad \alpha=1,2,
$$

where $\mu=\mu^{\mathrm{N}} / \mu^{\mathrm{R}}$.

Suppose that the value of the crown stretch ratio $t$ is sufficiently small that $s \leqslant s_{\mathrm{a}}$ for all particles. The equations governing the corresponding inflated state are given by (16)(22) and (27). These are reduced to a system of differential equations for $\lambda_{1}, \lambda_{2}, \eta$ as follows. One equation is obtained by combining (15), (16) and (27) to give

$$
\frac{\partial \lambda_{1}}{\partial R}=\Lambda_{1}^{\mathrm{e}}\left(\lambda_{1}, \lambda_{2}, \eta ; R\right)
$$

where

$$
\Lambda_{1}^{\mathrm{e}}\left(\lambda_{1}, \lambda_{2}, \eta ; R\right)=\frac{\lambda_{1}}{\lambda_{2}} \frac{\eta\left(\lambda_{2}^{2}-\lambda_{1}^{2}\right)-\left(3 \lambda_{3}^{2}-\lambda_{1}^{2}\right)\left(\eta-\lambda_{2}\right)}{R\left(\lambda_{1}^{2}+3 \lambda_{3}^{2}\right)}
$$

with $\lambda_{3}$ given by (15).

A second equation is the compatibility relation obtained by eliminating $r$ between eqns (14) and (17),

$$
\frac{\partial \lambda_{2}}{\partial R}=\frac{\eta-\lambda_{2}}{R}=\Lambda_{2}^{\mathrm{e}}
$$

The third equation is obtained from eqns (18)-(20) as

$$
\frac{\partial \eta}{\partial R}=\Lambda_{3}^{\mathrm{e}}\left(\lambda_{1}, \lambda_{2}, \eta ; R\right)
$$

where

$$
\Lambda_{3}^{\mathrm{e}}\left(\lambda_{1}, \lambda_{2}, \eta ; R\right)=\frac{\eta}{\lambda_{1}} \frac{\partial \lambda_{1}}{\partial R}+\frac{\lambda_{3} \lambda_{1}\left(\lambda_{1}^{2}-\eta^{2}\right)}{R} \frac{\sigma_{2}}{\sigma_{1}}-Q \frac{\lambda_{1}}{\lambda_{3}} \frac{\left(\lambda_{1}^{2}-\eta^{2}\right)^{1 / 2}}{\sigma_{1}}
$$

together with eqns (15), (27), (33) and (34). The use of the superscript " $\mathrm{e}$ " indicates that the expression applies in regions of the membrane where the material remains elastic.

An alternative to eqns (35) and (36) is the non-dimensional version of (22),

$$
\eta= \pm\left[\lambda_{1}^{2}-\left(\frac{Q \lambda_{1}^{2} \lambda_{2}^{2} R}{2 \sigma_{1}}\right)^{2}\right]^{1 / 2}
$$

Boundary conditions are obtained as follows. Continuity of the deformed midsurface implies that $r=0$ and $\partial z / \partial R=0$ at $R=0$. Equations (13), (14) and (17) then give

$$
\lambda_{1}=\lambda_{2}=\eta=t \quad \text { at } \quad R=0 .
$$

Since the sheet is fixed at its outer boundary, 


$$
\lambda_{2}=1 \quad \text { at } \quad R=1 \text {. }
$$

The system of differential equations (32)-(36), together with boundary conditions (38) and (39) define a boundary value problem for $\lambda_{1}, \lambda_{2}$ and $\eta$ when $Q$ is specified. Its solution applies when $t$ is sufficiently small that $s(R, t) \leqslant s_{\mathrm{a}}$ for $R \in[0,1]$.

Next, suppose that the value of the crown stretch ratio $t$ is such that $s \geqslant s_{\mathrm{a}}$ over part of the deformed surface. As discussed in Section 3, this will be near the crown. The particles in this domain are undergoing conversion. The complement to this domain contains particles which are still unconverted. The radius of the interface between the converting and unconverted material is denoted by $R_{\mathrm{a}}$.

Equations (32)-(36) apply for $R \in\left[R_{\mathrm{a}}, 1\right]$. The equations for $R \in\left[0, R_{\mathrm{a}}\right]$ are derived in the same manner as were eqns (32)-(36), but with the constitutive equation given by eqn (31). Let the expression for $\sigma_{1}$ be substituted into the first term in eqn (16). The result can be written in the form

$$
\frac{\partial\left(\lambda_{3} \sigma_{1}\right)}{\partial R}=A_{1} \frac{\partial \lambda_{1}}{\partial R}+A_{2} \frac{\partial \lambda_{2}}{\partial R}+\lambda_{3} \frac{\partial \bar{I}}{\partial R}
$$

where $I$ denotes the integral in the expression for $\sigma_{1}$ given by eqn (31), and

$$
\begin{aligned}
& A_{1}=\frac{\lambda_{3}}{\lambda_{1}}\left\{-\sigma_{1}+2 b\left(\lambda_{1}^{2}+\lambda_{3}^{2}\right)-4 a s^{\prime}\left(\lambda_{1}^{2}-\lambda_{3}^{2}\right)^{2}\left[\left(I_{1}-3\right)+\lambda_{2}^{2}\left(I_{2}-3\right)\right]\right\} \\
& A_{2}=\frac{\lambda_{3}}{\lambda_{2}}\left\{-\sigma_{1}+2 b \lambda_{3}^{2}-4 a s^{\prime}\left(\lambda_{1}^{2}-\lambda_{3}^{2}\right)\left(\lambda_{2}^{2}-\lambda_{3}^{2}\right)\left[\left(I_{1}-3\right)+\lambda_{1}^{2}\left(I_{2}-3\right)\right]\right\} .
\end{aligned}
$$

$s^{\prime}$ denotes the derivative of the function defined in eqn (26) with respect to its argument.

Recall the definition of $\tau(R, \hat{s})$ as the inverse of $s(r, \hat{t})$ for fixed $R$. Thus, when $\hat{s}$ takes the value $s(R, t)$ in the integrand of $\bar{I}, \tau(R, s(r, t))=t$ and the integrand vanishes. It follows that

$$
\frac{\partial}{\partial R} \int_{s_{\mathrm{a}}}^{s} a(\hat{s})\left(\frac{\lambda_{1}^{2}}{\hat{\lambda}_{1}^{2}}-\frac{\lambda_{3}^{2}}{\hat{\lambda}_{3}^{2}}\right) \mathrm{d} \hat{s}=\int_{s_{\mathrm{z}}}^{s} a(\hat{s}) \frac{\partial}{\partial R}\left(\frac{\lambda_{1}^{2}}{\hat{\lambda}_{1}^{2}}-\frac{\lambda_{3}^{2}}{\hat{\lambda}_{3}^{2}}\right) \mathrm{d} \hat{s}
$$

The result of evaluating $\partial \bar{I} / \partial R$ can be written as

$$
\frac{\partial I}{\partial R}=\frac{\partial \lambda_{1}}{\partial R} A_{3}+\frac{\partial \lambda_{2}}{\partial R} A_{4}+A_{5},
$$

where

$$
\begin{gathered}
A_{3}=\frac{2}{\lambda_{1}} \int_{s_{\mathrm{a}}}^{s} a(\hat{s})\left(\frac{\lambda_{1}^{2}}{\hat{\lambda}_{1}^{2}}+\frac{\lambda_{3}^{2}}{\hat{\lambda}_{3}^{2}}\right) \mathrm{d} \hat{s}, \\
A_{4}=\frac{2}{\lambda_{2}} \int_{s_{\mathrm{a}}}^{s} a(\hat{s}) \frac{\lambda_{3}^{2}}{\hat{\lambda}_{3}^{2}} \mathrm{~d} \hat{s}, \\
A_{5}=-2 \int_{s_{\mathrm{a}}}^{s} a(\hat{s})\left[\left(\frac{\lambda_{1}^{2}}{\hat{\lambda}_{1}^{2}}+\frac{\lambda_{3}^{2}}{\lambda_{3}^{2}}\right) \frac{D \lambda_{1}}{\hat{\lambda}_{1}}+\frac{\lambda_{3}^{2}}{\hat{\lambda}_{3}^{2}} \frac{D \lambda_{2}}{\hat{\lambda}_{2}}\right] \mathrm{d} \hat{s},
\end{gathered}
$$

in which $D \lambda_{\mathrm{a}}$ is the total derivative of $\lambda_{\alpha}$ with respect to $R$ at a fixed value $\hat{s}$ of the deformation state parameter, 


$$
D \lambda_{\alpha}=D \lambda_{\alpha}(R, \tau(R, \hat{s}))=\lambda_{\alpha 1}(R, \tau(R, \hat{s}))+\lambda_{\alpha 2}(R, \tau(R, \hat{s})) \frac{\partial \tau(R, \hat{s})}{\partial R}
$$

$\lambda_{\alpha 1}$ and $\lambda_{\alpha 2}$ denote the partial derivative with respect to the first and second arguments, respectively.

Let eqn (40) and (44) be substituted into (16) and use be made of (32). This leads to

$$
\frac{\partial \lambda_{1}}{\partial R}=\Lambda_{1}^{\mathrm{c}}
$$

where

$$
\Lambda_{\mathrm{i}}^{\mathrm{c}}=\frac{\frac{\eta \lambda_{3}\left(\sigma_{1}-\sigma_{2}\right)}{R \lambda_{2}}-\frac{\left(A_{2}+A_{4}\right)\left(\eta-\lambda_{2}\right)}{R}-\lambda_{3} \frac{\partial \bar{I}}{\partial R}}{A_{1}+A_{3}},
$$

with $\sigma_{1}$ and $\sigma_{2}$ given by eqn (31). An expression for $\partial \eta / \partial R$ is obtained by substituting eqn (49) into (18) and again using (31). This expression is denoted by $\Lambda_{3}^{c}$. The superscript "c" refers to expressions which apply in regions where the material is "converting".

The boundary value problem now consists of the system of equations

$$
\begin{aligned}
& \frac{\partial \lambda_{1}}{\partial R}=\Lambda_{1}, \\
& \frac{\partial \lambda_{2}}{\partial R}=\frac{\eta-\lambda_{2}}{R}=\Lambda_{2}, \\
& \frac{\partial \eta}{\partial R}=\Lambda_{3} .
\end{aligned}
$$

$\Lambda_{i}$ is given by $\Lambda_{i}^{c}$ for $R \in\left[0, R_{\mathrm{a}}\right]$, the domain of conversion, and by $\Lambda_{i}^{\mathrm{e}}$ for the complementary domain, $\left[R_{\mathrm{a}}, 1\right]$, where the material is still unconverted. Note that $\Lambda_{2}^{\mathrm{e}}=\Lambda_{2}^{\mathrm{c}}=\Lambda_{2}$. Continuity of the solution is required at the interface between the domains. Boundary conditions (38) and (39) still apply.

\section{NUMERICAL METHOD OF SOLUTION}

The response of the membrane involves an interaction between the conversion process and the properties of the original and newly formed networks. The intention here is to focus on the conversion process. Consequently, it is assumed that $\mu^{\mathrm{N}}=\mu^{\mathrm{R}}$ in eqn (28), or $\mu=1$ in (31). With this choice, it can be shown (Huntley, 1992), that the material softens near $R=0$. This occurs because the scission of the original material network causes its contribution to the total stress to reduce faster than new contributions are produced by the increasing deformation of the newly formed networks.

The solution procedure described here is based on the expectation, as described in Section 4 , that $s(R, t)$ decreases monotonically with $R$, and hence that the membrane consists of a central region of converting material, $R \in\left[0, R_{\mathrm{a}}\right]$ and an outer region of unconverted elastic material.

The boundary value problem associated with each inflated state is solved numerically. The solution is found at radii denoted by $R_{j}, j=1,2, \ldots, I, R_{1}=0, R_{J}=1$, and at values of the crown stretch ratio denoted by $t_{k}$. Solutions are first obtained for values of $t_{k}$ at which the particles of the membrane remain elastic. After determining the value of the crown stretch ratio at which conversion is initiated, solutions are then obtained for larger values of $t_{k}$ corresponding to increasing conversion.

The method of solution for the elastic regime is discussed first. Let $t_{k}=\lambda_{1}\left(0, t_{k}\right)=\lambda_{2}\left(0, t_{k}\right)$ be sufficiently small that $s\left(0, t_{k}\right)<s_{\mathrm{a}}$. As it is assumed that the 
greatest deformation occurs at $R=0$, it follows that $s\left(R, t_{k}\right)<s_{\mathrm{a}}, R \in[0,1]$. A value of the dimensionless pressure $Q$ is assumed and the system of differential equations (32)-(36) is integrated using the second order Runge-Kutta method. The integration process is begun at $R_{1}=0$ using boundary condition (38) with $t=t_{k}$. The Runge-Kutta method uses the values of the right-hand sides of (32)-(36) at $R_{j}$ in order to calculate the solution at $R_{j+1}$. However, at $R_{1}=0$, the right-hand sides are undefined. This difficulty is resolved by the analysis of Adkins and Rivlin (1952), who showed that a necessary condition for the solution to be bounded at $R_{1}=0$ is that $\partial \lambda_{1} / \partial R=\partial \lambda_{2} / \partial R=\partial \eta / \partial R=0$ there.

A consequence is that the Runge-Kutta procedure, which is based on the incrementation of the unknowns by values calculated from these derivatives, cannot get started. This difficulty is avoided by using the alternative equation (37), with the positive sign. $\eta(R, t)$ is then expressed in terms of $\lambda_{1}(R, t)$ and $\lambda_{2}(R, t)$. With this relation, the problem is reduced to a system of equations for $\lambda_{1}$ and $\lambda_{2}$ given by (32)-(34). Using the Rungc-Kutta procedure, variable $R$ is incremented to the non-zero value $R_{2}$ and the other quantities are evaluated at $R_{1}=0$. This causes a change in $\eta$ from its value at $R_{1}=0$ which in turn allows the integration process to get started. One integration step is carried out using this reduced system. The full system (32)-(36) is used for the remaining steps, in order to allow automatically for the possibility that $\eta=\partial r / \partial R$ becomes negative at some radial node.

The computed value of $\lambda_{2}\left(1, t_{k}\right)$ is used to check boundary condition (39). If the boundary condition is not satisfied for the assumed value of $Q$, a new value is chosen by a secant iteration method and the numerical integration of (32)-(36) is repeated. This process is continued until $\left|\lambda_{2}\left(1, t_{k}\right)-1\right| \leqslant \varepsilon$, for a specified value of $\varepsilon$.

The method of solution when conversion occurs is discussed next. Now the crown stretch ratio $t$ has values $t_{k}$ for which $s\left(0, t_{k}\right)>s_{\mathrm{a}}$, and integrals appear in the right-hand sides $\Lambda_{i}$ in (51) owing to their presence in the expressions for $\sigma_{1}, \sigma_{2}, A_{3}, A_{4}, A_{5}$. Consider a specific radius $R_{j}$ and let the corresponding values of the deformation state parameter be denoted by $s_{k}$, where

$$
s_{k}=s\left(R_{j}, t_{k}\right)
$$

Note also that

$$
t_{k}=\tau\left(R_{j}, s_{k}\right)
$$

Equal increments $t_{k+1}-t_{k}$ need not correspond to equal increments $s_{k+1}-s_{k}$. Moreover, an increment $t_{k+1}-t_{k}$ need not correspond to the same increment $s_{k+1}-s_{k}$ at different radii $R_{j}$. Thus, the integrals are approximated by a Simpson's rule which allows for unequal increments in the integration variable $s$.

Recall the notation $\hat{\lambda_{\mathrm{a}}}=\lambda_{\mathrm{a}}(R, \tau(R, \hat{s}))$. A typical term in the integral becomes

$$
\int_{s_{k}}^{s_{k+2}} \frac{a(\hat{s})}{\lambda_{\alpha}^{2}} \mathrm{~d} \hat{s}=\int_{s_{k}}^{s_{k+2}} \frac{a(\hat{s})}{\lambda_{\alpha}^{2}\left(R_{j}, \tau\left(R_{j}, \hat{s}\right)\right)} \mathrm{d} \hat{s} \approx \sum_{l=k}^{k+2} \frac{a\left(s_{l}\right)}{\lambda_{\alpha}^{2}\left(R_{j}, t_{l}\right)} W_{l}
$$

where use is made of (53) and $W_{l}$ denote the weighting coefficients

$$
\begin{aligned}
& W_{1}=\left(\frac{-c^{2}+c+2}{6}\right) \delta \\
& W_{2}=\frac{(c+1)^{3}}{6 c} \delta \\
& W_{3}=\frac{\left(2 c^{2}+c-1\right)}{6 c}
\end{aligned}
$$

with $\delta=s_{k+1}-s_{k}, c=\left(s_{k+2}-s_{k+1}\right) / \delta$. 
Suppose that $s_{m}<s_{\mathrm{a}}$ and $s_{m+1}>s_{\mathrm{a}}$ for some value of $m$. Then the value $t_{\mathrm{a}}$ at which $s=s_{\mathrm{a}}$ for a particle $R_{j}$ lines between two nodes. Such a point is indicated by a triangle in Fig. 1. As the lower limit of the integrals is $s_{\mathrm{a}}$, it is necessary to find this value of $t_{\mathrm{a}}$. Let $P \lambda_{\alpha}\left(R_{j}, t\right)$ be a second degree polynomial in $t$ constructed by Lagrange interpolation through the points $\left(t_{k}, \lambda_{a}\left(R_{j}, t_{k}\right)\right), k=m-2, m-1, m . P \lambda_{\alpha}\left(R_{j}, t\right)$ is used as an approximation to $\lambda_{\alpha}\left(R_{j}, t\right)$ for $t \in\left[t_{m-2}, t_{m+1}\right]$. Let $P s\left(R_{i}, t\right)$ be a function of $t$ which is an approximation to $s\left(R_{j}, t\right)$ for $t \in\left[t_{m-2}, t_{m+1}\right]$. It is constructed by using $P \lambda_{\alpha}\left(R_{j}, t\right)$ in eqns (25) and (26). Then $t_{\mathrm{a}}$ is given by the solution to

$$
s_{\mathrm{a}}=P s\left(R_{j}, t_{\mathrm{a}}\right) .
$$

The value for stretch ratio $\lambda_{\alpha}\left(R_{j}, t_{\mathrm{a}}\right)$ is then given by $P \lambda_{\alpha}\left(R_{j}, t_{\mathrm{a}}\right)$.

Consider integration from $s_{\mathbf{a}}$ to $s\left(R_{j}, t_{k}\right)$, for some value of $k$. If there is an even number of increments in this integration interval, Simpson's rule approximation (54) is used over each pair of increments. If there is an odd number of increments, the integral from $s_{\mathrm{a}}$ to the next largest value of $s$ is approximated using a trapezoidal rule. The remaining integral is over an even number of intervals and is approximated as before.

Now consider the system of eqns (51) for some specific value of the crown stretch ratio, $t_{K}>t_{\mathrm{a}}$. It is assumed that since the conversion process was initiated at $R=0$, continued inflation has caused all particles within some radius $R_{\mathrm{a}}$ to undergo the conversion process. For each $R \in\left[0, R_{\mathrm{a}}\right], \Lambda_{1}$ and $\Lambda_{3}$ contain integrals. These integrals can be approximated by finite sum expressions which contain $\lambda_{\alpha}\left(R, t_{\mathrm{a}}\right), \ldots, \lambda_{\alpha}\left(R, t_{k}\right), \ldots, \lambda_{\alpha}\left(R, t_{K}\right)$ and similar terms in $D \lambda_{\alpha}$ [see eqn (47)]. Suppose that $\lambda_{z}\left(R, t_{\mathrm{a}}\right), \ldots, \lambda_{\alpha}\left(R, t_{K-1}\right)$ and $D \lambda_{\alpha}\left(R, t_{\mathrm{a}}\right), \ldots$, $D \lambda_{\alpha}\left(R, t_{K-1}\right)$ have been previously determined. Then $\Lambda_{1}^{c}$ and $\Lambda_{3}^{c}$ depend on the unknown functions $\lambda_{\alpha}\left(R, t_{K}\right), \eta\left(R, t_{K}\right)$. They also depend on the variable $R$ both explicitly and through the dependence on $R$ of the previously determined solutions corresponding to $t_{k} \leqslant t_{K-1}$. Thus, a set of ordinary differential equations for $\lambda_{\alpha}\left(R, t_{K}\right), \eta\left(R, t_{K}\right)$ on $R \in\left[0, R_{\mathrm{a}}\right]$ is obtained. The set for $R \in\left[R_{\mathrm{a}}, 1\right]$ is given by eqn (51) with $\Lambda_{i}=\Lambda_{i}^{\mathrm{e}}$.

This system of equations for the entire domain is integrated using the second order Runge-Kutta method. This method is selected because evaluations are required only at the nodal points $R_{j}$, and not at intermediate points as is called for by the fourth order method. Recall that dependence on $R$ arises through the previously obtained solutions $\lambda_{\alpha}\left(R_{j}, t_{k}\right)$, $\eta\left(R_{j}, t_{k}\right), k<K$, at these nodal points. The evaluation of $\Lambda_{i}$ at $R_{j}$ uses these previously stored values.

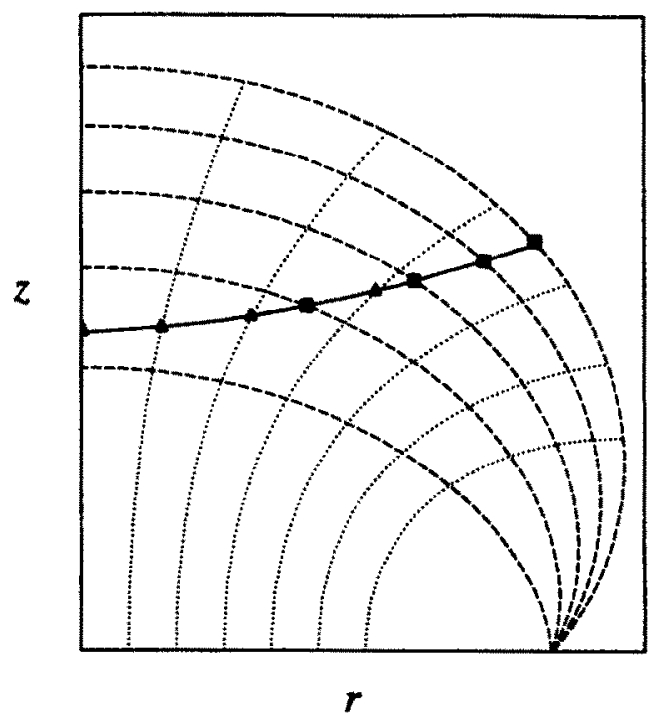

Fig. 1. Inflated profiles for increasing crown stretch ratios, showing the particle paths and conversion front. Triangles denote the intersections of the conversion front (solid line) with particle paths (dotted lines), rectangles denote intersections with profiles (dashed lines). 
The integration process beings at $R_{1}=0$ using boundary condition (38) with $t=t_{K}$. The $\Lambda_{i}$ involve expressions which are undefined at $R_{1}=0$. Since the Runge-Kutta method requires the evaluation of $\Lambda_{i}$ at the nodal points, the same difficulty arises which was discussed in conjunction with the solution method in the elastic regime. By an analysis similar to that of Adkins and Rivlin (1952), it can be shown that $\partial \lambda_{1} / \partial R=\partial \lambda_{2} / \partial R=\partial \eta / \partial R=0$ at $R_{1}=0$. The discussion provided in conjunction with the solution method in the elastic regime again applies here.

Suppose a solution for $\lambda_{\alpha}$ has been obtained at $R_{j}$. In order to proceed to $R_{j+1}$, it is first assumed that the particle at $R_{j+1}$ is undergoing conversion. The $\Lambda_{i}$ are evaluated at $R_{j+1}$ using the expressions for $\Lambda_{i}^{\mathrm{c}}$, which apply in the conversion regime. These use the stored histories $\lambda_{\alpha}$ and their derivatives at $R_{j+1}$, including the extrapolated approximations to the values at conversion $t_{\mathrm{a}}$, as solved from (56), and $\lambda_{x}\left(R_{j+1}, t_{\mathrm{a}}\right)=P \lambda_{x}\left(R_{j+1}, t_{\mathrm{a}}\right)$. The values of $\lambda_{\alpha}\left(R_{j+1}, t_{K}\right)$ are calculated using the Runge-Kutta method, and the corresponding value of the deformation state parameter is determined. Denote this value by $s^{\text {est }}\left(R_{j+1}, t_{K}\right)$. If $s^{\text {est }}\left(R_{j+1}, t_{K}\right) \geqslant s_{\mathrm{a}}$, the solution is accepted and the method proceeds to node $R_{j+2}$.

If $s^{\text {est }}\left(R_{j+1}, t_{K}\right)<s_{\mathrm{a}}$, it is assumed that the material at $R_{j+1}$ has not begun to convert. A new radial node is introduced at $R_{\mathrm{a}}=R_{j}+\Delta R, \Delta R<R_{j+1}-R_{j}$, to represent the radius $R_{\mathrm{a}}$ of the interface between the regions of the converting and unconverted material. Such a point is indicated by a square in Fig. 1. For a specified value of $\Delta R$, a Runge-Kutta step is taken using equations for the converting regime to evaluate the solution at $R_{\mathrm{a}}$. A secant iteration method is used to determine $\Delta R$ so that $\left|s\left(R_{i}+\Delta R, t_{K}\right)-s_{\mathrm{a}}\right| \leqslant \varepsilon$. For $R_{j} \in\left[R_{\mathrm{a}}, 1\right]$ the material is assumed to be elastic and the governing equations are given by eqn (51) with $\Lambda_{i}=\Lambda_{i}^{\mathrm{e}}$. These are integrated using the Runge-Kutta method, with continuity assumed at $R_{\mathrm{a}}$.

This section is concluded with a discussion of two numerical operations which occur in the computation of the various integrals. First consider $\partial \tau / \partial R$ which appears in eqn (48). It is evaluated at radius $R_{j}$ and deformation state parameter $s_{k}$ using the backward difference approximation

$$
\frac{\partial \tau}{\partial R}\left(R_{j}, s_{k}\right)=\frac{t_{j, k}-t_{j-1, k}}{R_{j}-R_{j-1}}
$$

where $t_{j, k}=t\left(R_{j}, s_{k}\right)$ and is the solution of

$$
s_{k}=P s\left(R_{j}, t_{k}\right)
$$

and $t_{i-1, k}=t\left(R_{j-1}, s_{k}\right)$ and is the solution of

$$
s_{k}=P s\left(R_{j-1}, t_{k}\right)
$$

Second, the value of $\lambda_{\alpha 2}=\partial \lambda_{\alpha} / \partial t$ appearing in eqn (48) is to be obtained at $R_{j}$ and $t_{k}$. This is calculated using the backward difference

$$
\lambda_{\alpha 2}\left(R_{j}, t_{k}\right)=\frac{P \lambda_{\alpha}\left(R_{j}, t_{k}\right)-P \lambda_{\alpha}\left(R_{j}, t_{k-1}\right)}{t_{k}-t_{k-1}}
$$

\section{NUMERICAL EXAMPLE}

In order to develop a numerical example, specific choices must be made for two material properties : the deformation state function in eqn (26) and the rate of conversion function $a(s)$ introduced in (5). The form of the deformation state function is chosen as

$$
s=\left[\left(I_{1}-3\right)^{2}+\left(I_{2}-3\right)^{2}\right]^{1 / n} .
$$

According to eqn (61), $s$ is constant on a circle in the $I_{1}-I_{2}$ plane. This curve in the $I_{1}-I_{2}$ 
plane is selected because it is characterized by a single parameter, the radius, as opposed to, say, a straight line which requires specification of its intercept with the $I_{1}$ axis and its slope.

As seen from eqns (15) and (25), the radius of the circle increases as $t^{8}$ near $R=0$. As $t$ becomes large, the radius increases very rapidly. If $n=2$, for example, $s$ also grows very rapidly and the increments in $t$ must become extremely small in order to control computational error. This difficulty is avoided by selecting $n=8$, so that $s$ increases as $t$.

The rate of conversion function is chosen to be quadratic on a finite domain,

$$
a(s)= \begin{cases}0, & s \in\left[0, s_{\mathrm{a}}\right), \\ \alpha\left(s-s_{\mathrm{a}}\right)\left(s-s_{\mathrm{c}}\right), & s \in\left[s_{\mathrm{a}}, s_{\mathrm{c}}\right], \\ 0, & s \in\left(s_{\mathrm{c}}, \infty\right) .\end{cases}
$$

According to this definition of $a(s)$ the process of material conversion occurs as the deformation state parameter $s$ increases over a finite interval and the process terminates when $s>s_{\mathrm{c}}$. Since the deformations under consideration are finite, the parameter $s$ will not exceed some finite value. Thus, $s_{\mathrm{c}}$ can be chosen sufficiently large that, in the present example, the conversion process need not reach completion. [It should be noted that other choices of $a(s)$ can be made in which $s_{\mathrm{c}}$ is unbounded.] Let the total volume fraction of material which may ultimately convert be denoted by $C$, where $C \leqslant 1$. Then, recalling eqn (7),

$$
C=\int_{s_{\mathrm{a}}}^{s_{\mathrm{c}}} a(s) \mathrm{d} s
$$

It then follows from eqns (62) and (63) that

$$
\alpha=-\frac{6 C}{\left(s_{\mathrm{c}}-s_{\mathrm{a}}\right)^{3}}
$$

Values of $s_{\mathrm{a}}, s_{\mathrm{c}}$ and $C$ are selected so as to bring out the differences in response between the membrane undergoing conversion and a neo-Hookean membrane (no conversion, $C=0$ ). Recall that $t$ is the control parameter in the numerical simulation, and consider the relation between the pressure $Q$ and the crown stretch ratio $t$ for a neo-Hookean membrane, shown in Fig. 2. The local maximum in the pressure occurs at $t=2.20$. Conversion is

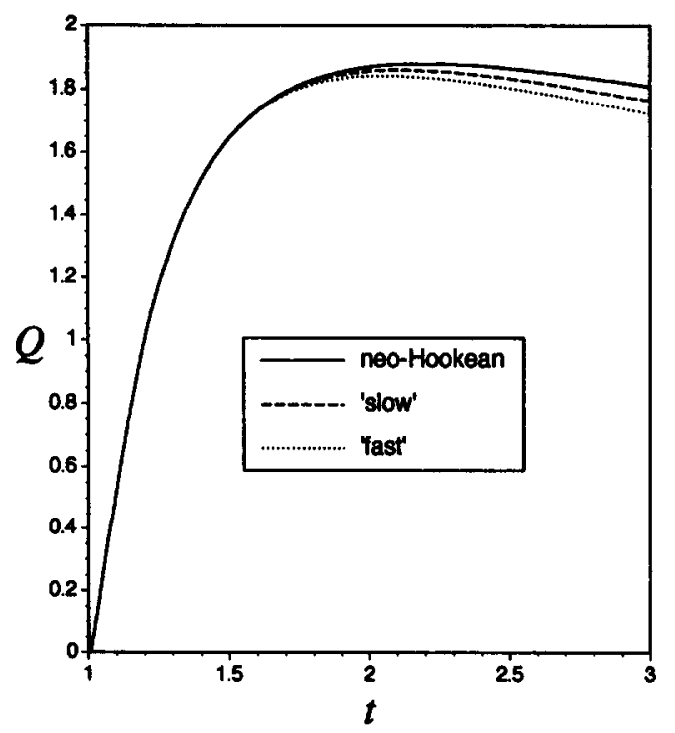

Fig. 2. Pressure vs crown stretch ratio, for neo-Hookean, "slow" conversion and "fast" conversion. 
specified to commence at the particle at the crown of the membrane when $t=1.5$. This enables the influence on the local maximum of the pressure to be observed. The value of the deformation state parameter at the particle at the crown corresponding to this choice of $t$ is $s_{\mathrm{a}}$. It follows from eqns (15), (25), (61) and $\lambda_{1}=\lambda_{2}=t=1.5$ that $s_{\mathrm{a}}=1.358$.

For many choices of $s_{\mathrm{c}}$ and $C$, application of the numerical procedure discussed in Section 5 has shown that the deformation state parameter $s$ decreases for some particles when $t$ becomes sufficiently large. That is, these particles undergo reversal of deformation. For particles which remain elastic (no conversion) this poses no difficulties. However, when there is conversion at a particle, new complexities arise as indicated in conjunction with eqn (10). Thus, values of $s_{\mathrm{c}}$ and $C$ are selected so as to allow simulation to be carried out over what is considered a reasonably broad range of deformations without the occurrence of reversal of deformation. Accordingly, $C=0.25$.

The simulation is carried out to $t=3.0$. Let $t_{\mathrm{c}}$ denote the stretch ratio of the particle at the crown if the conversion process there were carried to completion. The corresponding value of the deformation state parameter is $s_{\mathrm{c}}$. As a first case, $t_{\mathrm{c}}=3.1$ and $s_{\mathrm{c}}=3.089$ are selected. Thus, at $t=3.0$, the volume fraction of the material converted at the crown, by (15), (25), (61)-(64), and $\lambda_{1}=\lambda_{2}=t$, is $0.99 \mathrm{C}$. For the purpose of comparison, a second case is considered with $t_{\mathrm{c}}=4.1$ and $s_{\mathrm{c}}=4.096$. When $t=3.0$, the corresponding volume fraction of material converted at the crown is $0.64 \mathrm{C}$. For convenience in discussing the results, the first case is referred to as "fast" conversion and the second as "slow" conversion.

Equal radial increments $\Delta R=0.01$ and crown stretch ratio increments $\Delta t=0.01$ are used in the calculations.

The figures show results for three cases : no conversion (pure neo-Hookean response), "slow" conversion and "fast" conversion. Figure 2 shows plots of pressure versus crown stretch ratio. Note that the pressure has a local maximum in each case. The maximum is reduced and occurs at smaller stretch ratios as conversion occurs faster. Recall that when a particle undergoes conversion, the stress required to maintain a state of deformation is reduced, i.e. the response softens. The plots show this effect. When some of the particles of the membrane are undergoing conversion, their stiffness is reduced and less inflation pressure is required to produce a specified stretch ratio at the crown. When the conversion process occurs over a smaller range of stretch ratios, i.e. occurs "faster", material stiffness reduces faster and therefore the required pressure is further reduced.

Inflated membrane profiles at a crown stretch ratio $t=3.0$ are shown in Fig. 3. This stretch ratio is produced in smaller inflated shapes as conversion occurs "faster". Figure 4 shows the increase in interface radius with the crown stretch ratio. The radius increases very rapidly as $t$ increases from the value $t_{\mathrm{a}}=1.5$ at initial conversion. It then approaches

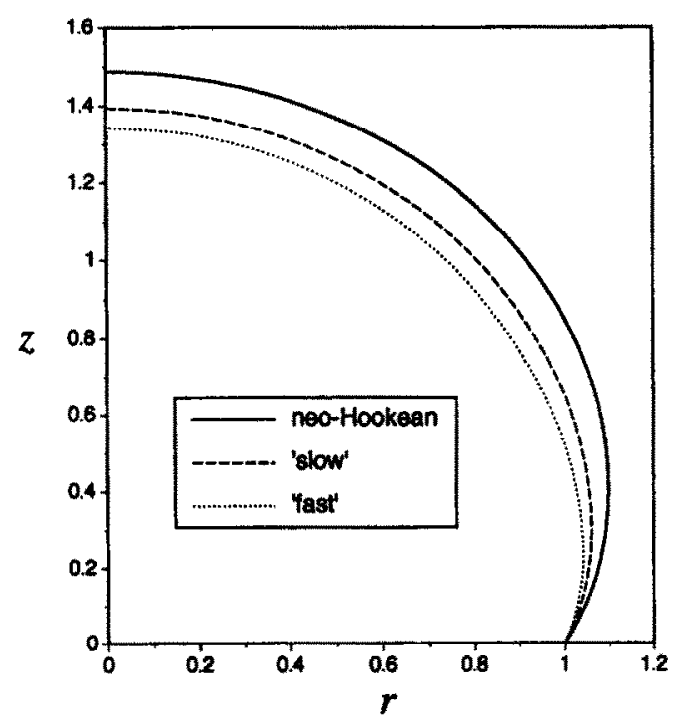

Fig. 3. Inflated profiles at a crown stretch ratio of $t=3.0$, for neo-Hookean, "slow" conversion and "fast" conversion. 


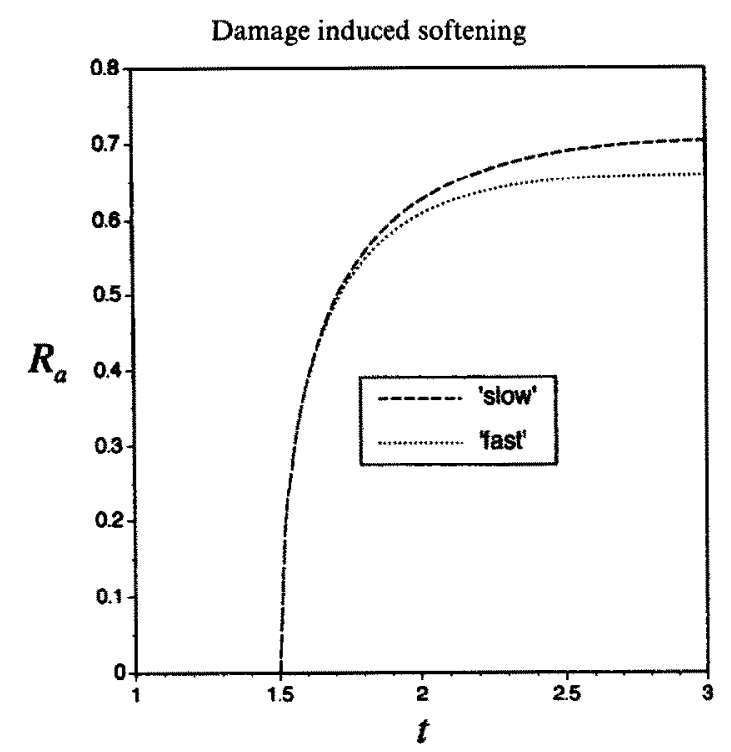

Fig. 4. Interface radius vs crown stretch ratio, for "slow" conversion and "fast" conversion.

a limiting value as $t$ approaches $t_{\mathrm{c}}$. This limit interface radius is larger when conversion occurs "slower". That is, there is a larger conversion zone when the conversion is "slower".

The reason for the results shown in Fig. 3 can be determined from the plots of the meridional and circumferential stretch ratios versus radius, in Figs 5 and 6, respectively. Plots are shown for $t=1.8$, just after initiation of conversion, and for $t=3.0$. The stretch ratios decrease from the crown to the support. At each particle undergoing conversion $R \in\left[0, R_{\mathrm{a}}\right]$, the stretch ratios are less than they would be were the membrane material to remain neo-Hookean. Therefore, the inflated profiles are smaller. Note that the unconverted particles $R \in\left[R_{\mathrm{a}}, 1\right]$ are at smaller stretch ratios than if there were no conversion. The material within the conversion region stretches more easily because of the softening effect associated with conversion. The response is softest near the crown. The specified crown stretch ratio thus occurs in the softer converting material in the inner core while the unconverted material in the outer layer is still at lower stretch ratios. This is seen in the stretch ratio distributions for $t=1.8$, at which the interface radius is 0.5452 for "fast" conversion and 0.5551 for "slow" conversion. This effect is seen to be even stronger when $t=3.0$. The interface radius is 0.6602 for "fast" conversion and 0.7043 for "slow"

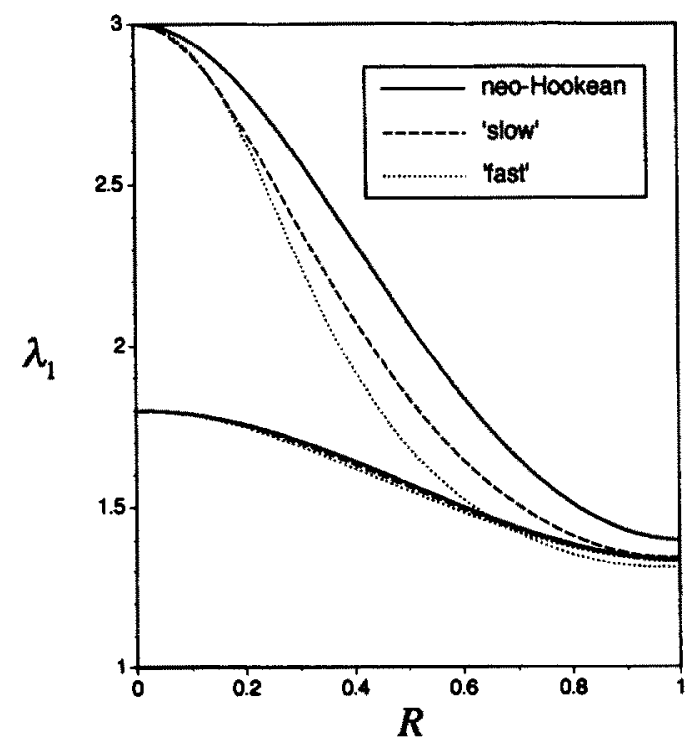

Fig. 5. Meridional stretch ratio vs $R$ at $t=1.8$ and $t=3.0$, for neo-Hookean, "slow" conversion and "fast" conversion. 


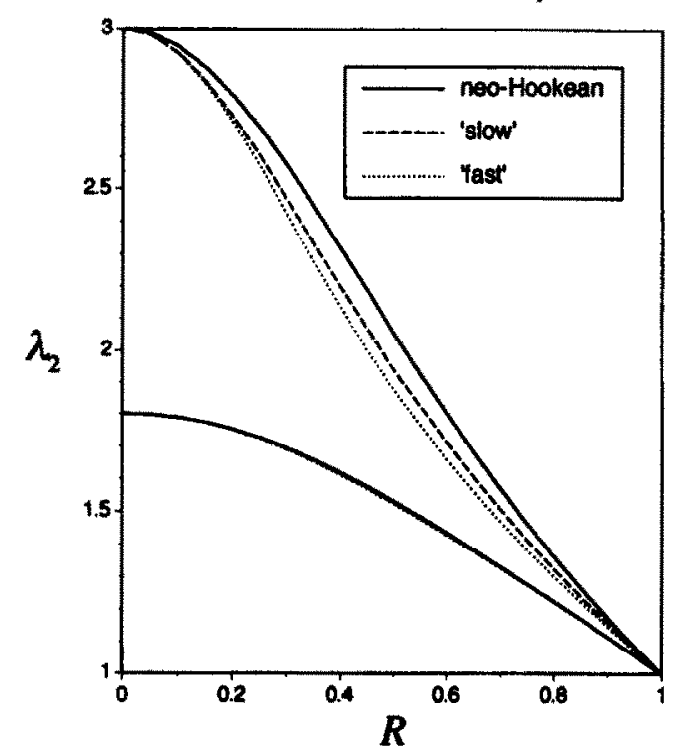

Fig. 6. Circumferential stretch ratio vs $R$ at $t=1.8$ and $t=3.0$, for neo-Hookean, "slow" conversion and "fast" conversion.

conversion. As $t$ increases from $t_{\mathrm{a}}$, the material near the crown undergoes increased conversion and softening of response. This occurs to such an extent that the stretch ratios of these particles reach the specified crown stretch ratio accompanied by reduced stretch ratios at the outer particles. Thus, in Fig. 5, consider the particles at radii greater than 0.7 . For the case of fast conversion, the stretch ratios at these particles become smaller at $t=3.0$ than at $t=1.8$. This suggests the evolution of a deformation reversal zone near the outer support, which spreads to meet the converting zone. When conversion is "faster", this reversal zone spreads faster.

Figures 7 and 8 , respectively, show plots of meridional and circumferential stresses versus crown stretch ratio for various radii. The stresses for the non-conversion (neoHookean) case increase monotonically with $t$ at each radius. This increase is diminished by conversion. At $R=0$, the increase is monotonic, although slower. At $R=0.4$, the increase is still monotonic. However, the rate of increase is substantially reduced as conversion occurs faster. Note that, at $R=0.8$, the meridional stress in the converting material has a local maximum and then begins to decrease. This occurs at particles in the outer region

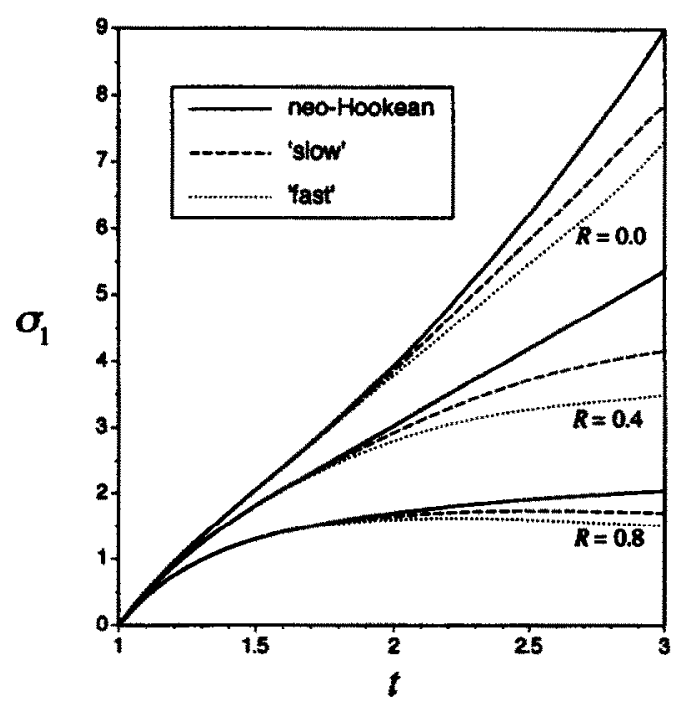

Fig. 7. Meridional stress vs crown stretch ratio for neo-Hookean, "slow" conversion and "fast" conversion at several radii. 


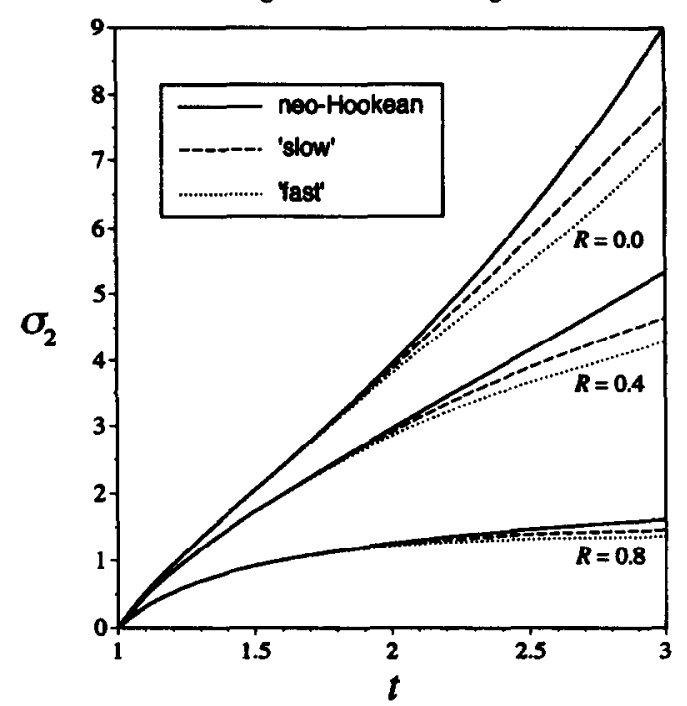

Fig. 8. Circumferential stress vs crown stretch ratio for neo-Hookean, "slow" conversion and "fast" conversion at several radii.

which remain elastic. As observed above, the stretch at these particles reduces, and by eqn (27), so does the stress.

In summary, these results show that conversion can have significant implications. The softening of the more highly stretched material can allow redistribution of deformation throughout the membrane and recovery of deformation of the stiffer unconverted material. This possibility will be explored in future work.

\section{REFERENCES}

Adkins, J. E. and Rivlin, R. S. (1952). Large elastic deformations of isotropic materials, part IX. Phil. Trans. A244, 505.

Green, A. E. and Adkins, J. E. (1960). Large Elastic Deformations. Oxford University Press, Oxford.

Hart-Smith, L. J. (1966). Elasticity parameters for finite deformations of rubber-like materials. Z. Angew. Math. Phys. 5, 608-626.

Hart-Smith, L. J. and Crisp, J. D. C. (1967). Large elastic deformations of thin rubber membranes. Int. J. Engng Sci. 5, 1-24.

Huntley, H. (1992). Applications of a constitutive equation for microstructural changes in polymers. Ph.D. Dissertation, University of Michigan.

Rajagopal, K. R. and Wineman, A. S. (1992). A constitutive equation for nonlinear solids which undergo deformation induced microstructural changes. Int. J. Plasticity 8, 385-395.

Treloar, L. R. G. (1944). Strains in an inflated rubber sheet, and the mechanism of bursting. Inst. Rubber Industry Trans. 19, 201.

Wineman, A. S. (1976). Large axisymmetric inflation of a nonlinear viscoelastic material by lateral pressure. Trans. Soc. Rhenl. 20, 203-225.

Wineman, A. S. and Rajagopal. K. R. (1990). On a constitutive theory for materials undergoing microstructural changes. Arch. Mech. 42, 53-75.

Wineman, A., Wilson, D. and Melvin, J. W. (1979). Material identification of soft tissue using membrane inflation. J. Biomech. 12, 841-850. 